\title{
Astrophysical jets: Observations, numerical simulations, and laboratory experiments
}

\author{
P. M. Bellan, ${ }^{1}$ M. Livio, ${ }^{2}$ Y. Kato, ${ }^{3}$ S. V. Lebedev, ${ }^{4}$ T. P. Ray, ${ }^{5}$ A. Ferrari, ${ }^{6}$ P. Hartigan, ${ }^{7}$ \\ A. Frank, ${ }^{8}$ J. M. Foster, ${ }^{9}$ and P. Nicolaï ${ }^{10}$ \\ ${ }^{1}$ Caltech, Pasadena, California 91125, USA \\ ${ }^{2}$ Space Telescope Science Institute, Baltimore, Maryland 21218, USA \\ ${ }^{3}$ University of Tsukuba, Ibaraki 3058577, Japan \\ ${ }^{4}$ Blackett Laboratory, Imperial College, London SW7 2BW, United Kingdom \\ ${ }^{5}$ Dublin Institute for Advanced Studies, 5 Merrion Square, Dublin 2, Ireland \\ ${ }^{6}$ Dipartimento di Fisica, Università di Torino, via Pietro Giuria 1, 10125 Torino, Italy \\ and Department of Astronomy and Astrophysics, University of Chicago, Chicago, Illinois 60637, USA \\ ${ }^{7}$ Department of Physics and Astronomy, Rice University, Houston, Texas 77251-1892, USA \\ ${ }^{8}$ Department of Physics and Astronomy and Laboratory for Laser Energetics, University of Rochester, \\ Rochester, New York 14627, USA \\ ${ }^{9} A W E$ Aldermaston, Reading RG7 $4 P R$, United Kingdom \\ ${ }^{10}$ Centre Lasers Intenses et Applications, Université Bordeaux 1-CEA-CNRS, 33405 Talence, France
}

(Received 13 June 2008; accepted 9 October 2008; published online 22 April 2009)

\begin{abstract}
This paper provides summaries of ten talks on astrophysical jets given at the HEDP/HEDLA-08 International Conference in St. Louis. The talks are topically divided into the areas of observation, numerical modeling, and laboratory experiment. One essential feature of jets, namely, their filamentary (i.e., collimated) nature, can be reproduced in both numerical models and laboratory experiments. Another essential feature of jets, their scalability, is evident from the large number of astrophysical situations where jets occur. This scalability is the reason why laboratory experiments simulating jets are possible and why the same theoretical models can be used for both observed astrophysical jets and laboratory simulations. (c) 2009 American Institute of Physics.
\end{abstract}

[DOI: $10.1063 / 1.3101812]$

\section{INTRODUCTION}

In the first chapter of his book Cosmic Plasma, ${ }^{1}$ Alfvén noted that in the past most theoretical physicists looked down on experimental plasma physics as a field which was too complicated and awkward and instead it was thought that with a limited amount of work the highly developed kinetic theory of ordinary gases could be extended to include ionized gases. He stated the following: "the theories were mathematically elegant and claimed to derive all properties of a plasma from first principles. In reality this was not true. Because of the complexity of the problem, a number of approximations were necessary which were not always appropriate. The theories had very little contact with experimental plasma physics; all awkward and complicated phenomena which had been observed in the study of discharges in gases were simply neglected."

Alfvén went on to argue for the importance of reconciling observations, theoretical models, and laboratory experiments and especially the notion that the plasma can often be considered as behaving like an electric circuit. In the second chapter of Cosmic Plasma he advocated that cosmic plasmas often have a filamentary (i.e., collimated) structure due to the self-pinching of electric currents and that plasmas often develop a cellular (i.e., heterogeneous) structure with different physics in the different cells and important effects going on at the boundaries between cells.

All these philosophical issues raised by Alfvén are very relevant to the study of astrophysical jets: one cannot de- velop an understanding of jets from observations alone, from theory alone, or from laboratory experiments alone and furthermore one cannot understand jets by assuming that the plasma is homogeneous. Jets appear to be both complicated and simple: observations show that on the one hand, jets are inhomogeneous and cannot be described by a simple extension of ordinary hydrodynamics; yet on the other hand, observations also show a great morphological similarity between jets having vastly different scales suggesting that there should be some simple underlying unifying concepts characterizing jets. Alfvén's proposed strategy of coordinating observation, modeling, and laboratory experiment is beginning to happen in the study of astrophysical jets. Because no individual or group can realistically pursue all three approaches in this strategy, to put the strategy into effect it is critical to establish good communication between the individuals and groups working on the different approaches.

The jet session at the HEDLA meeting contains ten talks on observation, theory, and laboratory experiments relating to astrophysical jets and so provides a means for effecting the coordination of the three approaches listed above. Just as Alfvén advocated, this linking of the three approaches is resulting in significant progress. It should be noted that the laboratory experiments are divided into two distinct types: (i) experiments where the jet is magnetohydrodynamically driven and (ii) experiments where the jet is purely hydrodynamic. Numerical modeling exists for both types. It is believed that the central engines driving astrophysical jets are probably magnetohydrodynamic (MHD), but far from the 
engine, MHD physics might become relatively unimportant so that the jet can be described by hydrodynamics alone.

This paper consists of short summaries of each of the ten talks in the order in which they were given. This order alternates observation, numerical modeling, and laboratory experiment.

\section{SUMMARIES OF THE TALKS}

Livio (Space Telescope Science Institute) began the session by giving a contextual overview of astrophysical jets. He emphasized that highly collimated jets are observed in many classes of astrophysical objects, ranging from active galactic nuclei and gamma-ray burst sources to young stellar objects (YSOs) and supersoft x-ray sources. The similarity of these jets having such widely varying parameters suggests that it is reasonable to assume that the mechanism for acceleration and collimation is the same in most if not all of the different classes of objects that exhibit jets. By invoking a primarily phenomenological approach, one can attempt to determine the constraints such an assumption of universality leads to. Using these constraints it is found that the most promising mechanism for the production of jets is an accretion disk threaded by a reasonably ordered, poloidal, largescale magnetic field. If the collimation is mainly poloidal (i.e., the magnetic flux is largest at the outer disk) rather than by hoop stresses, then only objects for which the ratio of the outer-to-inner disk radii is large can produce highly collimated jets. Finally, it is argued that truly powerful jets are produced by systems in which on top of an accretion disk threaded by a vertical field, there exists an additional source of heat or wind, possibly associated with the central object. The role of the black hole spin (in those systems in which the accretor is a black hole) remains ambiguous.

Kato (University of Tsukuba) described recent threedimensional (3D) numerical MHD simulations of astrophysical jets. The emergence of a magnetic tower in magnetized accretion flows revealed by these global MHD simulations provides an important clue for understanding the jet launching mechanism. ${ }^{2,3}$ The magnetic interaction of magnetic loops connecting differentially rotating objects appears to be a promising mechanism for launching jets in an accreting system. Although observational resources have not yet resolved the launching mechanism of astrophysical jets, plasma experiments confirm the formation of magnetic towers. If the magnetic tower mechanism is responsible for launching jets in any accreting system, difficult questions arise because not every accreting system has jets. Hence, one can ask the following: what are the conditions for launching astrophysical jets? The aim of the presentation talk was to try to answer this question.

3D MHD simulations of astrophysical jets indicate that at least three ingredients are necessary for launching jets, namely, (i) external pressure outside the disk, (ii) sufficiently strong magnetic fields, and (iii) appropriate magnetic field topology. The stronger the external pressure, the weaker the jet becomes while the stronger the field strength, the stronger the jet becomes. However, magnetic field thresholds depend on topology and, in particular, the polarity and coherence

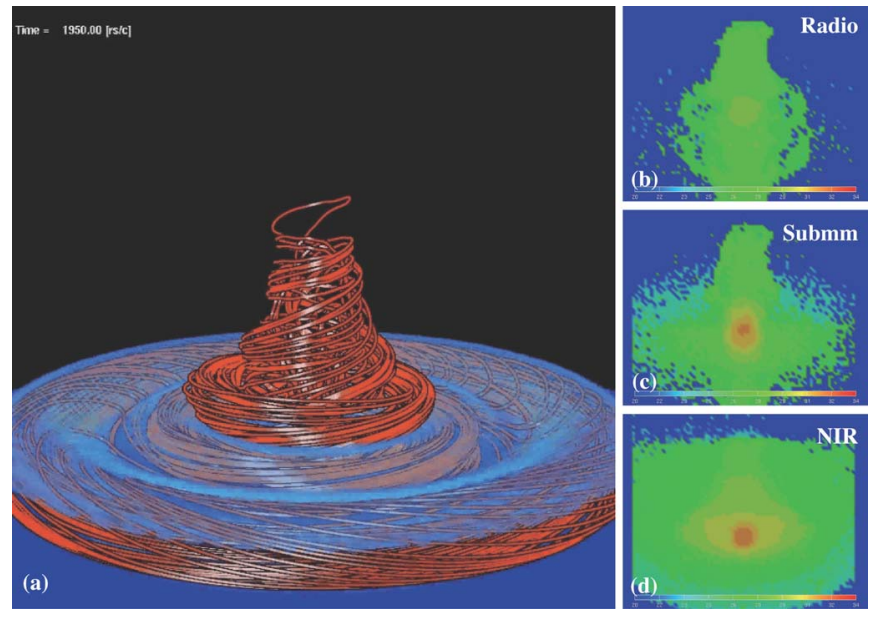

FIG. 1. (Color online) A snapshot of 3D MHD simulations of astrophysical jets and three images of $3 \mathrm{D}$ radation transfer simulations of the same 3D MHD data. (a) The emergence of a magnetic tower in magnetized accretion flows around black holes. Magnetic field lines and the isosurface of the density are shown by red solid lines and light-blue surface, respectively. (a) (b), (c), and (d) show synthesis images in radio, submillimeter, and nearinfrared bands, respectively.

length may affect the disk dynamo. Recent microquasar observations may provide some hints to answer these questions. Fender $\mathrm{et} \mathrm{al}^{4}$ found that when the spectrum is dominated by nonthermal emission, mildly relativistic steady jets are observed. On the other hand, when the spectrum is dominated by thermal emission, no jets are observed. Remarkably, highly relativistic transient jets are observed during the transition between these two distinct states. This observation indicates that more than one type of accretion disk can be the launch pad of astrophysical jets.

Since radiative processes are now realized to be key physics issue affecting the transition of accretion flows, the future study of astrophysical jets requires a radiation transfer calculation in magnetized accretion flows. Some preliminary simulations have been conducted for 3D MHD accretion flows and jets where 3D radiation transfer is taken into account. Figure 1(a) shows simulations of a magnetic tower jet and Figs. 1(b)-1(d) show synthesis images in radio, submillimeter, and near-infrared wavelength bands, respectively.

Lebedev (Imperial College) presented experiments on the formation of magnetically driven supersonic radiatively cooled plasma jets on the MAGPIE pulsed power facility (1 MA, $240 \mathrm{~ns}$ ). The jets are driven by the pressure of the toroidal magnetic field ${ }^{5,6}$ in topology similar to the "magnetic tower" model of astrophysical jets. ${ }^{7,8}$ The generated outflow consists of a current-currying central jet surrounded by a magnetically dominated cavity, which in turn is confined by the pressure of ambient medium. The experimental configuration was modified in comparison with previous work $^{5,6}$ and used radial $\mathrm{Al}$ foils instead of radial wire arrays, which allowed generation of several episodes of the magnetic tower jet eruptions (Fig. 2). The subsequent magnetic bubbles have higher propagation velocities $(150-300 \mathrm{~km} / \mathrm{s})$ and catch up with previously ejected material, producing shocks. The start of each episode is accompanied by an x-ray burst from the central jet compressed by the magnetic field. 


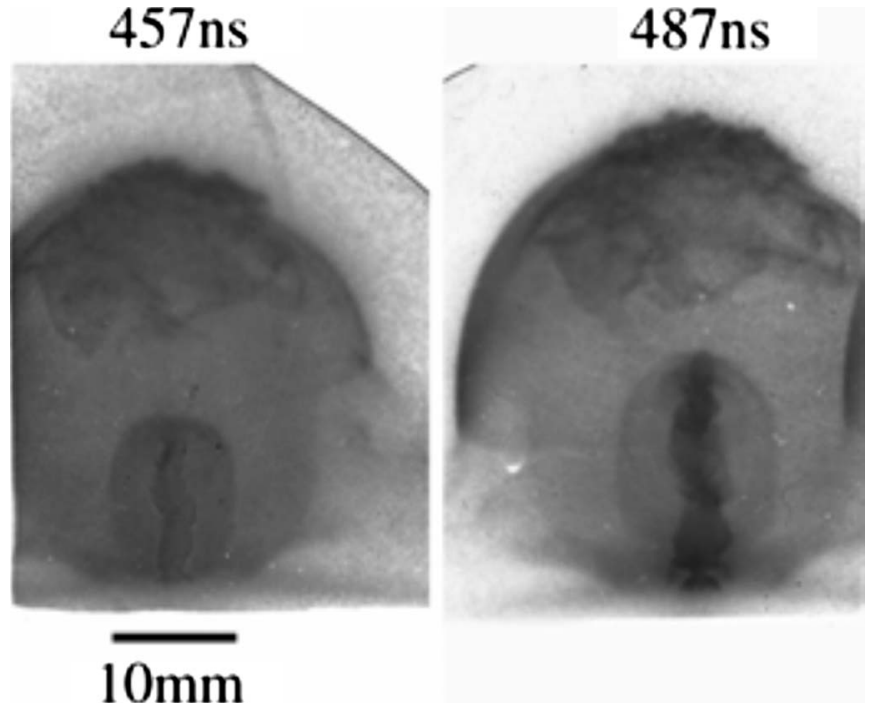

FIG. 2. XUV emission images showing the magnetic tower with the central jet on axis, evolving within remnants of previously ejected outflow.

These experiments suggest that periodic formation of magnetic tower jets in astrophysical situations could be responsible for some of the observed variability of astrophysical jets. It is believed that the experiments are scalable to astrophysical flows because critical dimensionless numbers such as the plasma collisionality, radiative cooling parameter $(\chi \sim 0.1)$, plasma beta $(\beta \sim 1)$, and magnetic Reynolds number $\left(\operatorname{Re}_{M} \sim 200\right)$ are all in astrophysically appropriate ranges. Measurements of the magnetic fields indicate that the toroidal magnetic flux is trapped in the cavities produced by the earlier episodes, and this could contribute to the collimation of the subsequent outflows. Measurements of plasma velocity and mass distribution, combined with the voltage and current measurements, allowed investigating the efficiency of the conversion of the Poynting flux energy into the kinetic energy of the outflow. The associated numerical simulations are performed with the 3D resistive MHD code GORGON. ${ }^{5,9}$

Ray (Dublin Institute for Advanced Studies) discussed launching mechanisms of astrophysical jets. He noted that jets from YSOs are the most striking phenomenon associated with star formation. ${ }^{10}$ Not only are these jets highly supersonic and well collimated but they can propagate for several parsecs, i.e., even beyond their parent cloud. ${ }^{11}$ As in the case of other jets, their velocities $\left(\approx 100-300 \mathrm{~km} \mathrm{~s}^{-1}\right)$ are close to the escape velocity of their source. The outflow phenomenon not only looks dramatic but could also be fundamental to the star formation process itself. In particular, jets from young stars may be responsible for

(1) dispersing a significant fraction of the natal stellar envelope,

(2) transporting angular momentum away from the accretion disk, thereby allowing accretion to occur, ${ }^{12}$ and

(3) supplying turbulence, and hence support, to the parent cloud (see Ref. 13 and this paper)

Moreover the phenomenon extends to objects with masses well below the stellar threshold: i.e., collimated outflows have recently been discovered from young brown dwarfs. ${ }^{14}$
Since jet emission derives from shock cooling zones, a large number of lines, both permitted and forbidden, are available for diagnostic purposes. One can thus readily derive fundamental parameters such as total density (neutral and ionic), temperature, mass loss rates, and cooling lengths with reasonable accuracy. ${ }^{15-17}$ In combination with multiepoch imaging, all the fundamental parameters necessary for laboratory simulations can, in principle, be determined, such as Mach number, opening angle of the flow, and Reynolds number. The only poorly defined parameters are the strength and direction of the magnetic field. There are, however, some indirect estimates of magnetic field strength based on emission line ratios and magnetized shock models. ${ }^{16}$ The inferred values are relatively low at several thousand a.u. from the source, implying that the plasma $\beta$ is high. These low inferred values are consistent with the absence of magnetic nose cones in YSO jets. ${ }^{18}$ Much closer to the source (i.e., $\leqslant 100$ a.u.) $\beta$ is expected to decrease ${ }^{19}$ and become closer to unity. This trend is consistent with the only direct measurement of the magnetic field strength in a YSO outflow from gyrosynchrotron emission observations ${ }^{20}$ and underlines the likely importance of the magnetic field in collimating YSO jets. $^{21,22}$ It is hoped that the next generation of radio telescopes (e.g., e-MERLIN and e-VLA) with their much greater sensitivity will detect further examples of nonthermal emission from YSO outflows. If so, such observations will supply the last missing parameter necessary for modeling the generation and focusing of these jets.

Ferrari (University of Torino and University of Chicago) also discussed issues relating to the launching of stellar jets. He reported tests of the validity of the Blandford and Payne model for launching jets from accretion disks using the FLASH and PLUTO numerical (nonrelativistic) $2.5 \mathrm{D}$ high resolution MHD codes. Steady-state numerical simulation models could be obtained provided that the disk resistivity is relatively high and the magnetic field is near equipartition. The range of the microphysical parameters is relatively small, in apparent contrast to observations which show that nature produces jets associated with very different astrophysical objects having extremely different physical characteristics. Non-steady-state solutions are, however, possible outside these ranges and give rise to intermittent or transient events, especially of magnetic tower type.

Plots of density and poloidal magnetic field in two steady-state solutions have been obtained by assuming large resistivity; these are shown in Fig. 3. Figure 3 (left) corresponds to initial magnetic field below equipartition and to a well-collimated jet. Figure 3 (right) corresponds to magnetic fields above equipartition and the collimated jet is accompanied by an equally strong extended wind. The flow does not cross the Alfvén surface within the integration domain and yields intermittent mass outbursts.

Figure 4 shows numerical results from a series of tests performed to understand the process of jet launching in the presence of a magnetized star at the center of the accretion disk. The interaction of the star's magnetosphere and the accretion disks can give rise to a different outflow configuration. Nevertheless it appears that jets are in all cases produced by the star; the disk may produce extended outflows 

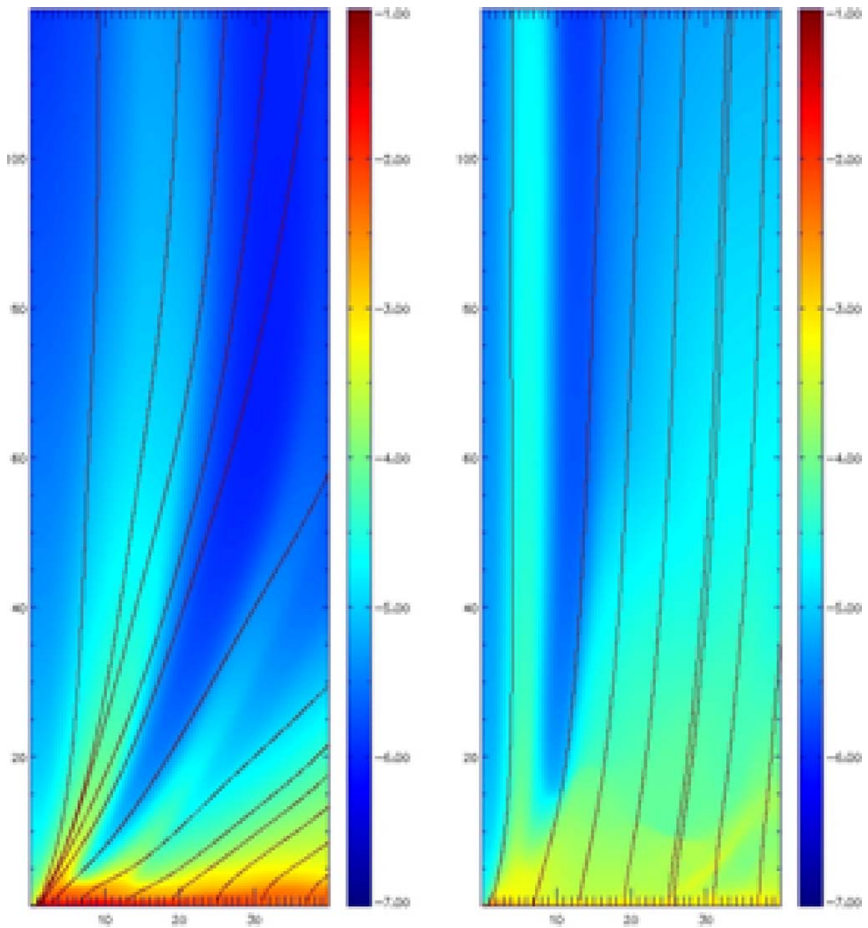

FIG. 3. (Color online) Left: initial magnetic field below equipartition yielding a well-collimated jet. Right: magnetic fields above equipartition; the collimated jet is accompanied by an equally strong extended wind (simulation by Ferrari, Zanni, and Tzerefacos).

without collimation that, in specific conditions, may be capable of extracting angular momentum from the system and so slow down the forming star. At the same time, intermittent coronal mass-ejection-type outflows can occur along the interface between the star and the disk open field lines.

The time evolution of an accretion disk interacting with a stellar magnetosphere having low resistivity and viscosity
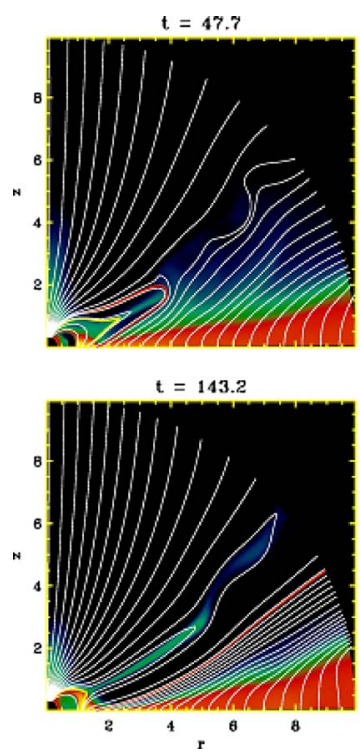
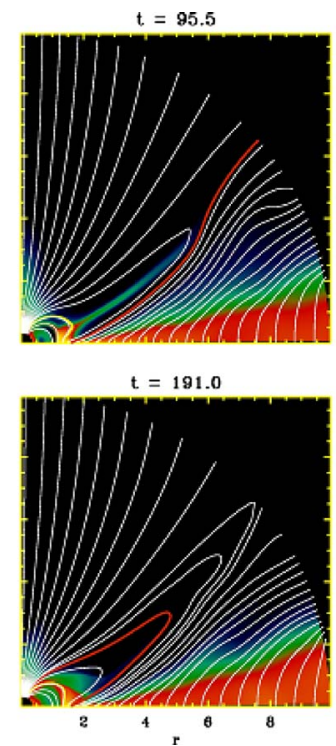

FIG. 4. (Color online) Jet launching in the presence of a magnetized star at the center of the accretion disk for the case of star's spin axis aligned with the disk's angular momentum vector (simulation by Ferrari, Zanni, and Tzerefacos).

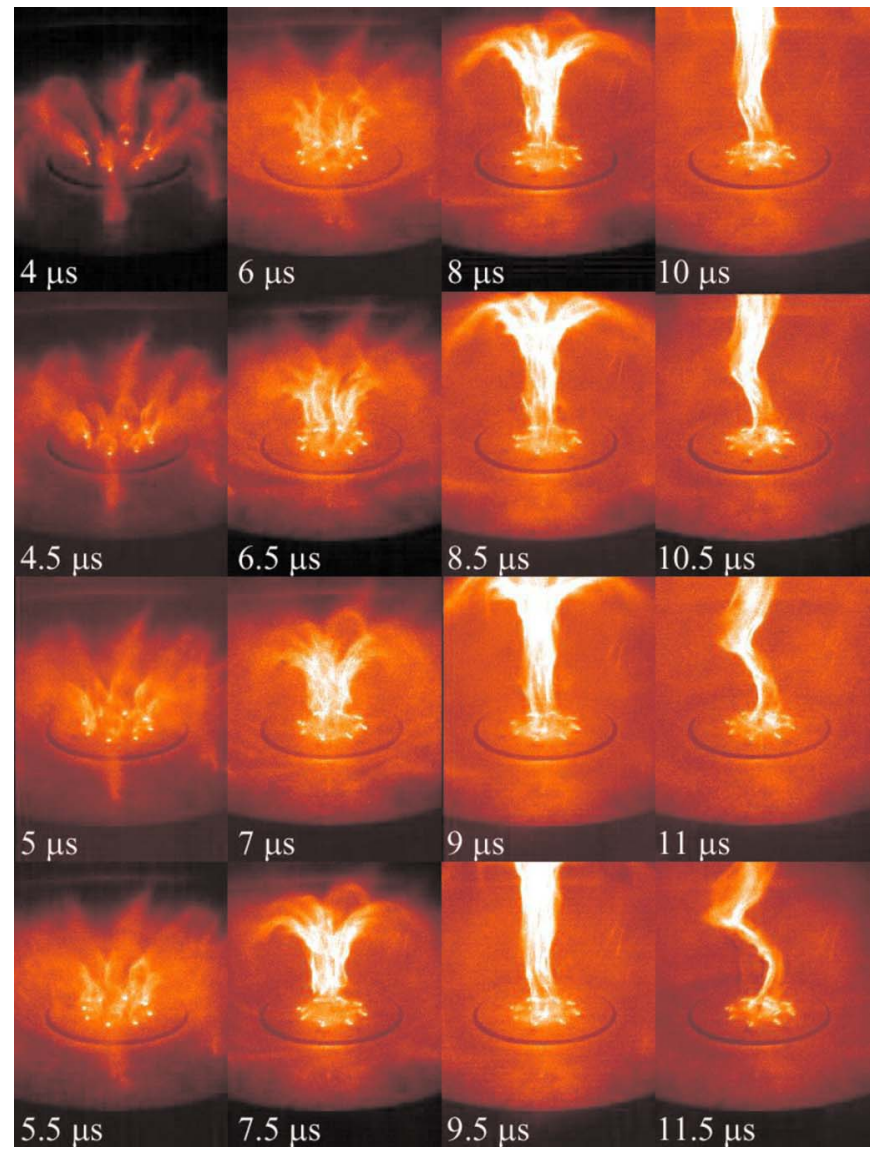

FIG. 5. (Color online) Dynamical evolution of laboratory simulation of astrophysical jet (from Ref. 23); magnetic pressure due to toroidal magnetic field inflates poloidal field resulting in magnetic tower configuration. Collimation results from pileup of frozen-in toroidal flux near the top. At later stages, the jet undergoes kink instability.

and Prandtl number around unity has been investigated for the case of the star's spin axis aligned with the disk's angular momentum vector. Outflows occur from both star and disk open field lines. The separation region between the two types of lines gives rise to intermittent mass ejections triggered by reconnection processes. Outflow from open magnetospheric field lines and intermittent matter ejections yield angular momentum loss from the star.

Bellan (Caltech) presented recent results on laboratoryproduced MHD plasma jets; ${ }^{23}$ a typical laboratory jet evolution is shown in Fig. 5. These highly reproducible jets have $150 \mathrm{kA}$, lengths of $20 \mathrm{~cm}$, and duration of $10 \mu \mathrm{s}$. They can be considered as magnetic tower jets whereby the pressure of the toroidal magnetic flux (due to a poloidal current) inflates a poloidal magnetic field.

The dynamical process can be explained starting from the dual concepts underlying MHD that (i) parallel currents attract each other while antiparallel currents repel each other and (ii) magnetic flux is frozen into the frame of the plasma. Concept (i) is phenomenologically equivalent to the MHD equation of motion while concept (ii) is phenomenologically equivalent to the induction equation. Bellan argued that in order to understand why collimation occurs, it is essential to take into account that magnetic flux is frozen into the frame of the plasma. Freezing in of flux is separately true for both 
toroidal and poloidal fluxes. The freezing of toroidal magnetic flux into the plasma frame means that the toroidal magnetic flux is carried along (i.e., convected) by a moving plasma such as a MHD-driven jet. If the jet velocity varies so that faster portions of the jet catch up with slower portions in a shocklike fashion, then not only will there be a pileup of plasma as faster plasma runs into slower plasma, but there will also be a pileup of toroidal magnetic flux as the toroidal magnetic flux frozen into the faster plasma runs into the toroidal magnetic flux frozen into the slower plasma. This piling up is characterized mathematically by the fluid velocity having a negative divergence, i.e., a convergence. The pileup of frozen-in toroidal magnetic flux causes an increase in the toroidal flux density which is just the toroidal magnetic field responsible for pinching. Thus, the pileup results in increased pinching force and so a reduction in jet radius. This provides collimation of the jet. ${ }^{23,24}$

Hartigan (Rice University) gave an overview of the observed hydrodynamical properties of stellar jets. He pointed out that jets from young stars are in many ways ideal for studying supersonic fluid dynamics. These remarkable collimated flows consist of multiple bow shocks which form in response to velocity perturbations, and cases also exist where jets deflect from obstacles located along the path of the flow and produce spectacular shocked wakes and shear. The hot gas behind the shocks in stellar jets radiates optically thin forbidden lines, from which one can measure turbulent linewidths, densities, ionization fractions, and temperature and even watch how the jets and shocks evolve in real time.

A series of laboratory experiments is underway at the Omega laser facility to follow shock wave formation and evolution in jets. Figure 6 (right) shows the result of an experiment where a supersonic jet is deflected from an obstacle in the flow. The resulting shocked spray is visually similar to Fig. 6 (left), a photograph of the HH 110 stellar jet deflecting from a dense molecular cloud. In both the experiment and the stellar jet the thermal conductivity and viscosity are unimportant, and the Mach numbers of the flows are comparable. In order to provide quantitative constraints on the behavior of the shocked spray, high spectral resolution observations of $\mathrm{HH} 110$ have been obtained with the $4 \mathrm{~m}$ telescope at Kitt Peak National Observatory. Observations presented in Fig. 7 clearly show that the linewidths of $\mathrm{H}$ alpha exceed those of the forbidden lines (S II) and (N II) along the flow. The thermal widths of the forbidden lines are unresolved with this instrument, so the observed velocity widths provide a direct measure of bulk motions along the line of sight for each knot, while the additional linewidth present in $\mathrm{H}$ alpha arises from thermal broadening. In this manner one can measure the postshock temperature, and therefore the shock velocities in the deflected jet.

Frank (University of Rochester) considered jets as heterogeneous systems. He noted that jets from all astrophysical objects are believed to be hypersonic systems of plasma ejected from a central source. The majority of models for these jets and outflows take the plasma to be essentially homogeneous. Any knots in the jet flow are assumed to be the result of pulsation in the flow speed at the injection point with the entire jet beam participating in the pulsation.

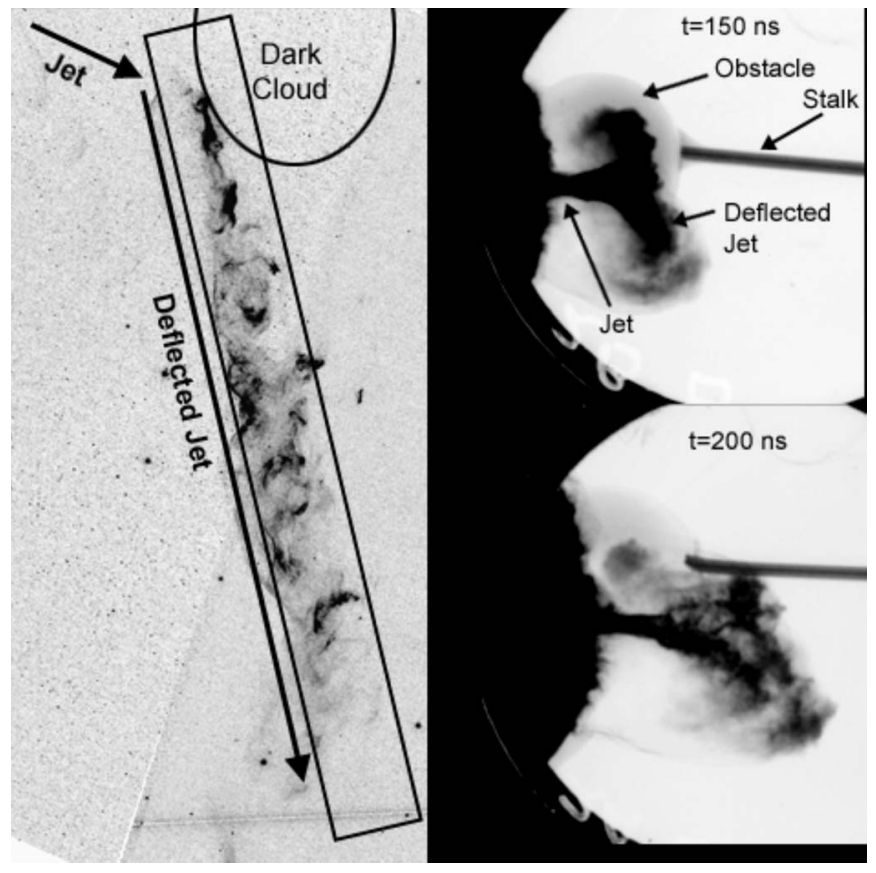

FIG. 6. Left: Hubble Space Telescope image of the stellar jet HH 110, which deflects from a dense dark cloud and exhibits a complex network of weak shock waves. Right: Radiograph of a deflected jet from the Omega laser facility. The jet enters from the left and is deflected by a spherical obstacle that is held in place by a stalk. The image taken at $200 \mathrm{~ns}$ has a developed structure that resembles that of $\mathrm{HH} 110$.

Using as inspiration recent MHD jet evolution experiments on pulse power machines, models are considered in which jets are essentially heterogeneous plasma systems. Kink-mode instabilities close to the jet launching disrupt but do not destroy the jet. The nonlinear saturation of the unstable modes fragment the beam into chains of dense knots that propagate at a range of velocities around the average beam speed.

The consequences of this model have been investigated using adaptive mesh refinement simulations. Results of these investigations show that many features of observed jets which have not previously been explained can be accounted for by taking into account the heterogeneity of the jet beam. In particular, the presence of subradial structure recovers the spur shocks and backward facing bows seen in high resolution HST observations of YSO jets.

Foster (AWE Aldermaston) described experiments with supersonic plasma jets at the Omega laser facility. He began by noting that large-scale directional outflows of supersonic plasma, also known as jets, are often encountered in astrophysics. These jets propagate through the interstellar medium which is often clumpy and where inhomogeneities affect the morphology of the shocks that are generated. The hydrodynamics is difficult to model as the problem is inherently $3 \mathrm{D}$, and the clumps are subject to a variety of fluid instabilities as they are accelerated and destroyed by shocks. Very largescale inhomogeneities may result in deflection of the jet itself. The traditional approach to understanding such phenomena is through theoretical analysis and numerical simulations. However, such numerical simulations have limited resolution, often assume axial symmetry, do not include all rel- 


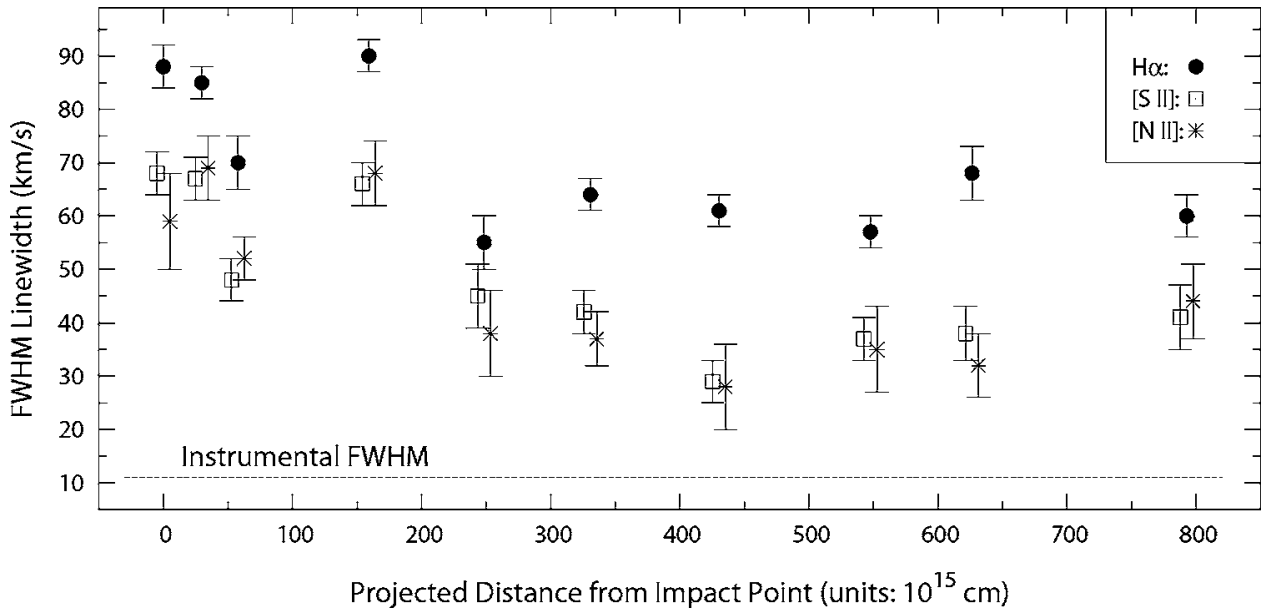

FIG. 7. Dynamics of the shocked spray in the HH 110 stellar jet. The emission linewidth of $\mathrm{H}$ alpha, which has both thermal and nonthermal components, exceeds that of the forbidden lines, where the thermal component is negligible. These observations constrain how the gas in the deflected jet is heated by weak shocks. evant physical processes, and may fail to scale correctly in Reynolds number and other key dimensionless parameters. They are frequently not tested by comparison with laboratory experiments.

Experiments carried out at the University of Rochester's Omega laser ${ }^{25}$ have investigated the physics associated with the propagation of plasma jets and shocks through both homogeneous $^{26,27}$ and inhomogeneous ${ }^{28}$ media. These experiments provide close analogs to structures observed in jets from young stars. Jets and shocks are created in experimental assemblies that are ablatively driven by a $190 \mathrm{eV}$ temperature "hohlraum" (which is itself heated by the Omega laser) and subsequently propagate into a low density hydrocarbonfoam medium (Fig. 8). The foam is either of uniformly low density $^{26}$ or contains localized (higher density) perturbations. ${ }^{28}$ Interaction of a jet with this fluid results in the development of a bow shock, and, in the case of a single density perturbation, results in deflection of the jet in a laboratory analog of the astrophysical object HH110. ${ }^{29-31}$ The

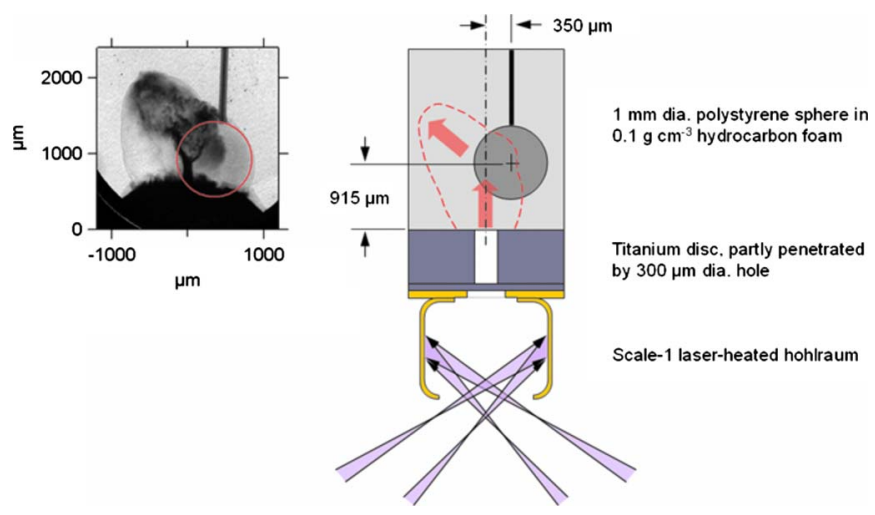

FIG. 8. (Color online) Configuration of the deflected-jet experiment and radiographic data. Beams of the Omega laser heat the gold hohlraum target, resulting in x-ray ablation of the surface of a titanium disc partly penetrated by a $300 \mu \mathrm{m}$ diameter hole. The directed outflow of shock-heated titanium from this hole results in a dense-plasma jet in a block of low density hydrocarbon containing a $1 \mathrm{~mm}$ diameter polystyrene sphere. The arrows indicate the incident and deflected jets, and the dashed line indicates the bow shock in the low density foam medium through which the jet propagates. The experimental data (left-hand figure) were obtained with a $9 \mathrm{keV}$ x-ray backlighting source at $200 \mathrm{~ns}$ after the onset of the laser drive. The red circle indicates the initial position of the sphere in the radiograph. hydrodynamic structures that develop in these experiments are revealed by x-ray "backlighting" radiography. These complex experimental data challenge both astrophysical and laser-plasma hydrodynamics computer codes.

Several radiation-hydrodynamics codes are used to model these experiments, including (primarily) the LANL/ SAIC and 3D adaptive mesh refinement code RAGE. ${ }^{32}$ Figure 9 shows synthetic radiographs obtained by postprocessing RAGE simulations of the experimental data of Fig. 8 for three levels of calculational resolution. Simulations at different resolutions clearly show differences in both small- and intermediate-scale structures. Several "benchmarks" are used for comparing the experiment and simulation including the position of the bow shock in the foam ahead of the jet, the mass of material in the jet (obtained from quantitative analysis of the radiographic images), and the power spectral density of optical depth (obtained from the discrete Fourier transform of the experimental and synthetic images) to quantify the small-scale structure in the potentially turbulent material resulting from deflection of the jet by the density perturbation. The existing experiments are well modeled by RAGE simulations.

Modeling will also be carried out using a new adaptive mesh refinement code called ASTROBEAR, ${ }^{33}$ developed for astrophysical applications, which can rapidly and accurately track the jet flows. ASTROBEAR utilizes high order accurate Riemann solvers and can run two-dimensional (2D) or 3D simulations. It now includes $\mathrm{MHD}$, ionization dynamics, $\mathrm{H}_{2}$ chemistry, radiative cooling (via cooling curves), as well as gravitational and radiative driving source terms.
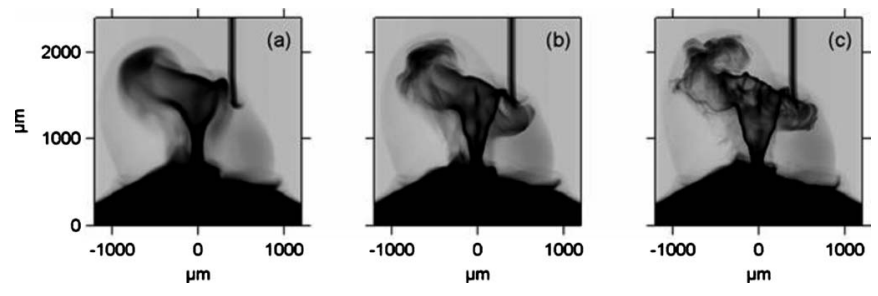

FIG. 9. The images are synthetic radiographs obtained by postprocessing the output of the adaptive mesh refinement code RAGE. The three simulations are for calculational resolutions of 12,6 , and $3 \mu \mathrm{m}$ [respectively, (a), (b), and $(\mathrm{c})]$. 


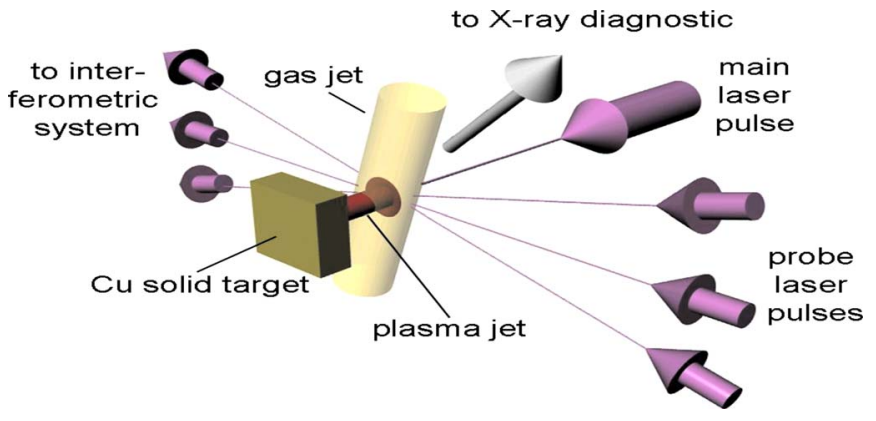

FIG. 10. (Color online) Experimental setup and diagnostics alignment.

Nicolaï (University of Bordeaux) described experiments with laser-driven plasma jets. A jet formation method developed recently ${ }^{34}$ allows the launching of a jet having a velocity around $400 \mathrm{~km} / \mathrm{s}$ and a density of $\sim 10^{18} \mathrm{~cm}^{-3}$ from a solid copper target using only a relatively small laser energy. Experimental, theoretical, and numerical studies have been conducted for the interaction of this fast jet with a gas placed in front of the target. By varying the gas density in the range $(0.2-8) \times 10^{18} \mathrm{~cm}^{-3}$ and the atomic composition (helium or argon) the relative density and the radiation cooling time can be controlled, and the transition from adiabatic to radiative interaction conditions can be investigated.

The experiment was carried out at the Prague Asterix Laser System iodine laser facility. ${ }^{35}$ A $0.438 \mu$ m laser beam having $290 \mathrm{~mm}$ diameter was focused by a $600 \mathrm{~mm}$ focal length aspherical lens onto a planar solid copper target which provided the plasma jet production. The laser energy was $\sim 100 \mathrm{~J}$, the focal spot radius on the target was $300 \mu \mathrm{m}$, and the pulse duration was $\sim 250 \mathrm{ps}$ (full width at half maximum). A secondary gaseous target was placed at a distance of $3.5 \mathrm{~mm}$ in front of a copper plate, see Fig. 10. A high pressure (2-40 bars) supersonic gas nozzle was employed to create a $3 \mathrm{~mm}$ diameter cylindrical column of argon or helium.

By using a combination of two complementary diagnostics in the optical and x-ray spectral range the main interaction zone structures were characterized. These structures were the density gradients marking the laser-induced gas ionization, the plasma jet launched from a solid target, the bow shock propagating through gas, the shocked gas behind the front, the contact discontinuity, and the shocked part of the copper plasma jet. Figure 11 presents typical shadowgrams and x-ray images for 10 bar argon and 20 bar helium gases. For argon gas there is a good correspondence between the shadowgram and the x-ray image, as both copper and argon are radiating $\mathrm{x}$ rays. For helium gas, the emission arises only from a copper jet, and the bow shock in helium is not visible.

The evolution of the interaction zone with time, the gas pressure, and material were compared with existing analytical estimates ${ }^{36}$ and detailed 2D radiation hydrodynamic simulations. ${ }^{37}$ In helium gas the radiation losses are small, the plasma behind the bow shock remains hot, and the thermal pressure prevents a strong compression. The interaction follows an adiabatic behavior. In the argon gas case, the strong radiation-losses induce formation of a dense, thin
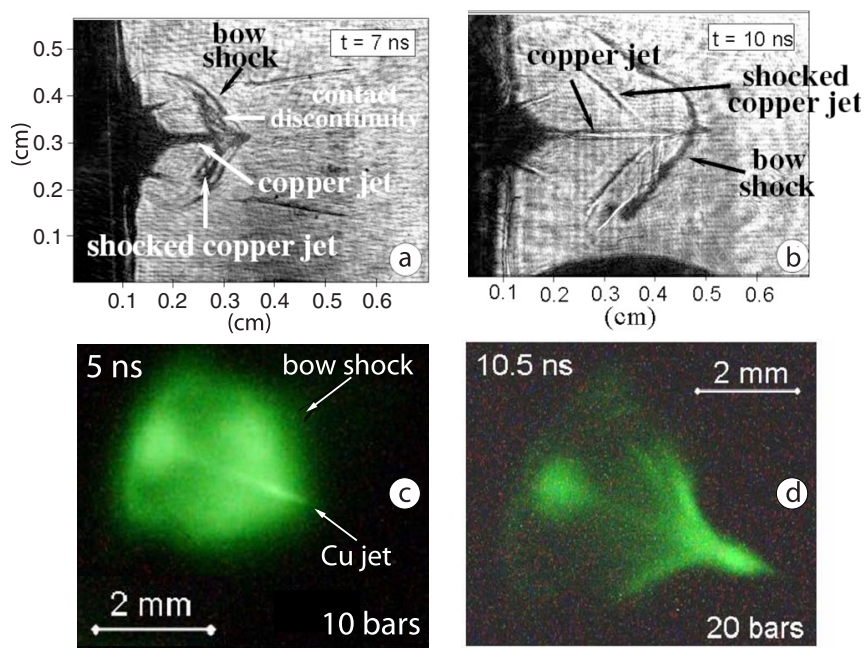

FIG. 11. (Color online) Shadowgrams (a) at $7 \mathrm{~ns}$ for the argon gas at 10 bars and (b) at $10 \mathrm{~ns}$ for the helium gas at 20 bars. X-ray images obtained in the same conditions for (c) argon gas and (d) helium gas.

shell. The observed behavior corresponds to a radiative cooling jet.

The structures observed in the experiment are qualitatively very similar to those seen in astrophysical objects having completely different time and space scales. ${ }^{38}$ Dimensional analysis shows that an appropriate scaling can be made to astrophysical jets seen in YSOs for the velocity, the Mach number, the jet-plasma density ratio, and the dissipative processes described by the cooling parameter. This latter can be tuned by varying the jet diameter, its velocity, the gas composition, and its density.

${ }^{1}$ H. Alfven, Cosmic Plasma (Reidel, Dordrecht, 1981).

${ }^{2}$ Y. Kato, S. Mineshige, and K. Shibata, Astrophys. J. 605, 307 (2004).

${ }^{3}$ Y. Kato, Astrophys. Space Sci. 307, 11 (2007).

${ }^{4}$ R. Fender, T. Belloni, and E. Gallo, Mon. Not. R. Astron. Soc. 355, 1105 (2004).

${ }^{5}$ A. Ciardi, S. V. Lebedev, A. Frank, E. G. Blackman, J. P. Chittenden, C. J. Jennings, D. J. Ampleford, S. N. Bland, S. C. Bott, J. Rapley, G. N. Hall, F. A. Suzuki-Vidal, A. Marocchino, T. Lery, and C. Stehle, Phys. Plasmas 14, 056501 (2007).

${ }^{6}$ S. V. Lebedev, A. Ciardi, D. J. Ampleford, S. N. Bland, S. C. Bott, J. P. Chittenden, G. N. Hall, J. Rapley, C. A. Jennings, A. Frank, E. G. Blackman, and T. Lery, Mon. Not. R. Astron. Soc. 361, 97 (2005).

${ }^{7}$ D. Lynden-Bell, Mon. Not. R. Astron. Soc. 369, 1167 (2006).

${ }^{8}$ D. Lynden-Bell, Mon. Not. R. Astron. Soc. 279, 389 (1996).

${ }^{9}$ J. P. Chittenden, S. V. Lebedev, C. A. Jennings, S. N. Bland, and A. Ciardi, Comments Plasma Phys. Controlled Fusion 46, B457 (2004).

${ }^{10}$ T. Ray, C. Dougados, F. Bacciotti, J. Eislöffel, and A. Chrysostomou, in Protostars and Planets V, edited by B. Reipurth, D. Jewitt, and K. Keil (University of Arizona Press, Tucson, 2007) p. 231.

${ }^{11}$ F. McGroarty and T. P. Ray, Astron. Astrophys. 420, 975 (2004).

${ }^{12}$ T. Ray and F. Bacciotti, Rev. Mex. Astron. Astrofis. 15, 206 (2003).

${ }^{13}$ Z. Y. Li and F. Nakamura, Astrophys. J. 640, L187 (2006).

${ }^{14}$ E. T. Whelan, T. P. Ray, F. Bacciotti, A. Natta, L. Testi, and S. Randich, Nature (London) 435, 652 (2005).

${ }^{15}$ F. Bacciotti and J. Eislöffel, Astron. Astrophys. 342, 717 (1999).

${ }^{16}$ P. Hartigan, Astrophys. Space Sci. 287, 111 (2003).

${ }^{17}$ S. Melnikov, J. Woitas, J. Eislöffel, F. Bacciotti, U. Locatelli, and T. P. Ray, Astron. Astrophys. 483, 199 (2008).

${ }^{18}$ A. Frank, D. Ryu, T. W. Jones, and A. Noriega-Crespo, Astrophys. J. 494, L79 (1998), Part 2. 
${ }^{19}$ P. Hartigan, A. Frank, P. Varniere, and E. G. Blackman, Astrophys. J. 661, 910 (2007), Part 1

${ }^{20}$ T. P. Ray, T. W. B. Muxlow, D. J. Axon, A. Brown, D. Corcoran, J. Dyson, and R. Mundt, Nature (London) 385, 415 (1997).

${ }^{21}$ J. Ferreira, C. Dougados, and S. Cabrit, Astron. Astrophys. 453, 785 (2006).

${ }^{22}$ F. H. Shu, D. Galli, S. Lizano, and M. J. Cai, in Star-Disk Interaction in Young Stars, Proceedings of the International Astronomical Union, IAU Symposium (2007), Vol. 243, pp. 249-264.

${ }^{23}$ S. You, G. S. Yun, and P. M. Bellan, Phys. Rev. Lett. 95, 045002 (2005).

${ }^{24}$ P. M. Bellan, Phys. Plasmas 10, 1999 (2003).

${ }^{25}$ J. M. Soures, R. L. McCrory, C. P. Verdon, A. Babushkin, R. E. Bahr, T. R. Boehly, R. Boni, D. K. Bradley, D. L. Brown, R. S. Craxton et al., Phys. Plasmas 3, 2108 (1996).

${ }^{26}$ J. M. Foster, B. H. Wilde, P. A. Rosen, R. J. R. Williams, B. E. Blue, R. F. Coker, R. P. Drake, A. Frank, P. A. Keiter, A. M. Khokhlov et al., Astrophys. J. 634, L77 (2005).

${ }^{27}$ J. M. Foster, B. H. Wilde, P. A. Rosen, T. S. Perry, M. Fell, M. J. Edwards, B. F. Lasinski, R. E. Turner, and M. L. Gittings, Phys. Plasmas 9, 2251 (2002).

${ }^{28}$ R. F. Coker, B. H. Wilde, J. M. Foster, B. E. Blue, P. A. Rosen, R. J. R. Williams, P. Hartigan, A. Frank, and C. A. Back, Astrophys. Space Sci. 307, 57 (2007).
${ }^{29}$ B. Reipurth, A. C. Raga, and S. Heathcote, Astron. Astrophys. 311, 989 (1996).

${ }^{30}$ A. Riera, R. Lopez, A. C. Raga, R. Estalella, and G. Anglada, Astron. Astrophys. 400, 213 (2003).

${ }^{31}$ E. M. D. Dal Pino, Astrophys. J. 526, 862 (1999), Part 1.

${ }^{32}$ M. L. Gittings, in Numerical Methods Symposium, 28-30 April 1992; Copies may be ordered from the Defence Nuclear Agency, 56801 Telegraph Road, Alexandria, VA 22310-3398.

${ }^{33}$ A. Poludnenko, P. Varniere, A. Cunningham, A. Frank, and S. Mitran, Lecture Notes in Computational Science and Engineering (Springer, Berlin, 2005), Vol. 41, pp. 331-340.

${ }^{34}$ A. Kasperczuk, T. Pisarczyk, S. Borodziuk, J. Ullschmied, E. Krousky, K. Masek, K. Rohlena, J. Skala, and H. Hora, Phys. Plasmas 13, 062704 (2006).

${ }^{35}$ K. Jungwirth, A. Cejnarova, L. Juha, B. Kralikova, J. Krasa, E. Krousky, P. Krupickova, L. Laska, K. Masek, T. Mocek et al., Phys. Plasmas 8, 2495 (2001).

${ }^{36}$ P. Drake, High Energy Density Physics (Springer, Berlin, 2006).

${ }^{37}$ P. H. Maire, R. Abgrall, J. Breil, and J. Ovadia, SIAM J. Sci. Comput. (USA) 29, 1781 (2007).

${ }^{38}$ E. M. D. Dal Pino, Fundamentals of Space Environment Science, Advances in Space Research Vol. 35 (Elsevier, Amsterdam, 2005), pp. 908-924. 